\title{
Impacto del programa juntos en las necesidades básicas insatisfechas, pobreza, salud y educación en los beneficiarios de la región Junín
}

\author{
Impact of the program together on unsatisfied basic needs, poverty, health and education in the \\ beneficiaries of the Junín region
}

\author{
Eugenia Fabián Arias ${ }^{1}$ (D) (8), Juan Carlos Pérez Ticse 2 (iD (8), Edith Rosana Huamán Guadalupe 3 (iD (8), \\ Luz María Vilcas Baldeón 4 (ID (8), Carmen Yudex Baltazar Meza 5 (iD) (8:)
}

\section{Cómo citar}

Fabián Arias, E., Pérez Ticse, J.C., Huamán Guadalupe, E. R., Vilcas Baldeón, L.M. y Baltazar Meza, C. Y. (2021). El impacto del programa juntos en necesidades básicas insatisfechas, pobreza, salud, y educación en la región Junín. Socialium, 5(2), 237-255. https://doi.org/10.26490/uncp.sl.2021.5.2.881

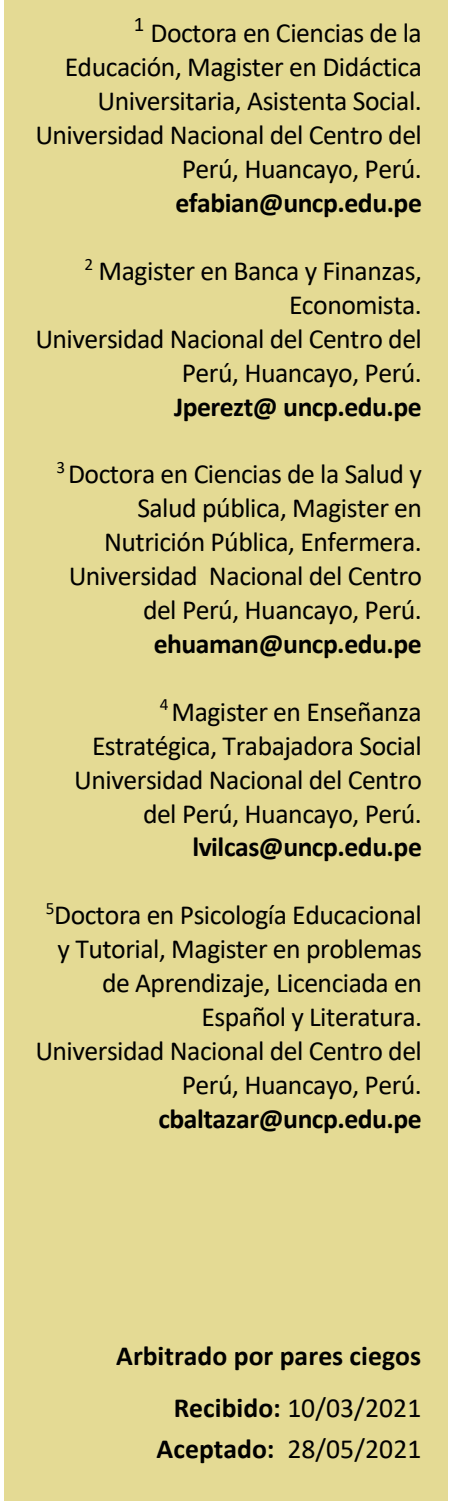

\section{RESUMEN}

El Programa Juntos, es un programa de transferencias monetarias condicionadas que se otorga un bono de S/200 soles bimensuales a hogares en situación pobreza y extrema pobreza. El objetivo del estudio fue determinar el impacto del Programa Juntos en las necesidades básicas insatisfechas, pobreza, salud y educación en la región Junín, tipo de investigación básico, nivel explicativo, carácter cuantitativo, diseño cuasi experimental, las unidades de análisis fueron los beneficiarios del programa juntos. La población fue 3990 hogares y la muestra para el tratamiento fue de 1459 hogares y para el grupo control 1,399 hogares del año 2015 al 2019; el método de investigación fue Diferencias en Diferencias con datos de las Encuestas Nacionales de Hogares, Demográfica y de Salud Familiar y procesado con el software Stata 15. Los resultados en necesidades básicas insatisfechas, generó un impacto de bajo nivel significativo en el tipo de vivienda y alumbrado por generador eléctrico ; en pobreza se evidenció un impacto de moderado nivel significativo; pero no se encontraron resultados significativos en los indicadores de salud, educación, la matrícula de niños y adolescentes generó un impacto moderadamente significativo ; en el idioma o lengua materna se evidenció un impacto positivo de bajo nivel significativo y en cuanto a la pensión mensual de enseñanza se observó un impacto negativo de moderado nivel significativo del programa. Se concluye que se encontraron algunos indicadores de bajo nivel significativo en necesidades básicas insatisfechas, pobreza y educación y no se encontró resultados significativos en salud.

Palabras clave: impacto; necesidades básicas; pobreza; salud; educación.

\section{ABSTRACT}

The Juntos Program is a conditional cash transfer program that grants a bimonthly S / 200 soles bonus to households living in poverty and extreme poverty. The objective of the study was to know the impact of the Juntos Program on unsatisfied basic needs, poverty, health and education in the Junín Region, type of basic research, descriptive level, quantitative character, quasi-experimental design, the analysis units were the beneficiaries schedule together. The population was 3,990 households and the treatment sample was 1,459 households and 1,399 households for the control group from 2014 to 2019; the research method was Differences in Differences with data from the National Household, Demographic and Family Health Surveys and processed with the Stata 15 software. The results in unsatisfied basic needs generated a significant low-level impact on the type of housing and lighting by electric generator; in poverty there was evidence of a moderately significant impact; but no significant results were found in the health indicators; in education the enrollment of children and adolescents generated a moderately significant impact; In the mother tongue or tongue, a positive impact of a low significant level was evidenced and regarding the monthly tuition pension, a negative impact of a moderately significant level of the program was observed. It is concluded that some indicators of low significant level were found in unsatisfied basic needs, poverty and education and no significant results were found in health.

Keywords: impact; basic needs; poverty; health; education. 


\section{Introducción}

El Programa de Transferencia Monetaria Condicionada "Juntos", es un programa nacional de apoyo directo a los más pobres el cual se inscribe dentro de la política social de lucha contra la pobreza. En el Perú el programa mencionado comenzó en el año 2005, teniendo como objetivo contribuir a la reducción de pobreza y con ello romper con la transmisión intergeneracional de la pobreza extrema, mediante la transferencia directa de dinero en efectivo para incentivar el acceso a los servicios de salud, nutrición y educación de las familias beneficiarias. Almeida et al. (2017)

A nivel internacional, el pionero del programa social fue "Progresa" en México, iniciado en 1997 para zonas rurales y modificado a partir del 2001 como "Oportunidades" que forma parte del programa nacional de desarrollo social 2001-2006; el programa "Bolsa escolar" de Brasil basado en pilotos municipales y estudios a nivel nacional. El éxito de adopción de estos programas se reflejó en 13 países. A nivel de América Latina fueron apoyados técnica y financieramente por el Banco Mundial (BM) y el Banco Internacional para el desarrollo (BID). Cardozo (2006)

En el Perú el Programa Social Juntos se creó en el 2005, dirigido a la población de mayor vulnerabilidad en situación de extrema pobreza por D.S.032-PCM-2000, adscrito a la presidencia del Consejo de Ministros (PCM) a través de la comisión interministerial de Agentes Sociales (CIAS). Se inició en 4 departamentos como es Ayacucho, Huánuco, Huancavelica y Apurímac, ampliándose progresivamente cada año en los demás departamentos, para los criterios de selección se tuvo en cuenta el Sistema de Focalización de Hogares (SISFOH), la participación de la comunidad y la participación de las autoridades. Aramburu (2010). El programa Juntos viene funcionando bajo determinadas condiciones para niños menores de 6 años de edad, mujeres embarazadas y en periodo de lactancia, que asisten a sus evaluaciones de salud de manera regular: Control, Crecimiento y Desarrollo (CRED) para niños. Para niños entre 6 y 19 años se exige asistencia escolar de por lo menos del 85 \% por año; y para mujeres embarazadas y madres en periodo de lactancia, controles prenatales y postnatales (vacunas contra el tétano, suplementos de hierro y ácido fólico y chequeos antiparasitarios). Los Beneficiarios del programa JUNTOS, son hogares o familias en condición de pobreza, especialmente de las zonas rurales, integradas por gestantes, niño, niña, adolescente y joven, hasta que culmine la educación secundaria o cumplan 19 años de edad. Así como otros programas de Transferencias Monetarias condicionadas, el programa juntos tiene dos grandes objetivos de corto y largo plazo; de corto plazo está orientado a reducir la pobreza proporcionando a los hogares transferencias monetarias y a largo plazo a romper la transmisión de la pobreza de generación en generación mediante la promoción del capital humano a través de acceso a la educación y servicios de salud. Perova y Vakis (2010). 
El objetivo principal del Programa social Juntos es contribuir a la reducción de la pobreza y evitar que los hijos sigan viviendo en la pobreza que afectó a sus padres y como propósito general generar capital humano dentro de los hogares en situación de pobreza extrema, en un marco de corresponsabilidad hogar-Estado, mediante la entrega de incentivos para el acceso y uso de servicios de salud, nutrición y educación. En la investigación realizada por (Perova y Vakis, 2010), revelan que los resultados cuantitativos encuentran impactos positivos en varias dimensiones de bienestar, tales como: la reducción de pobreza, mejora de ingresos, salud y educación. Sin embargo, la evaluación no encuentra impactos en indicadores finales como lo es la nutrición infantil, anemia y desarrollo cognitivo.

En el impacto del Programa Juntos en la región Junín, no existen evidencias científicas sobre el impacto en necesidades básicas insatisfechas, pobreza, salud y educación, por ello el objetivo del presente estudio fue evaluar el impacto del Programa Juntos en las necesidades básicas insatisfechas, pobreza, salud y educación. Considerando que el tema de estudio es muy relevante debido a que no se tiene monitoreo y evaluaciones del programa a nivel de la región Junín, encontrándose solo de otras regiones. Así mismo, se abordó el presente tema porque la mayoría de la población beneficiaria que participa en el Programa Juntos se encuentra en condición de pobreza y pobreza extrema que afecta el bienestar de los usuarios, pese a estar implementándose por más de 15 años, no se tiene evidencia científica sobre los logros de este programa. Porque no se tenía una línea de base debido a que no permitía estimar los efectos causales atribuibles al programa; por esa razón se ha tomado los datos del periodo del INEI-ENAHO (2015-2019) con el propósito de contribuir en la reformulación de los lineamientos de políticas de intervención del Programa Juntos y al mismo tiempo de emprender los monitoreos y evaluaciones tendientes a responder a las necesidades de la población beneficiaria.

En base a los índices de pobreza que presenta el país, los gobiernos han visto por conveniente implementar los programas sociales de apoyo a la población necesitada con el propósito de superar la pobreza presentada, cuyas características de algunos se tiene efectos positivos y negativos, sin embargo, cabe precisar que no se ha observado cambios sustanciales. En la investigación realizada por Perova y Vakis (2010), revela que los resultados cuantitativos encuentran impactos positivos en varias dimensiones del bienestar, tales como: la reducción de la pobreza, mejora de los ingresos económicos, salud y educación. Sin embargo, en la evaluación no se encuentra impacto en indicadores finales como la nutrición infantil, anemia y desarrollo cognitivo.

En el estudio de evaluación de impacto del programa nacional de apoyo directo a los más pobres (JUNTOS) en Collao, Puno, los resultados dan a conocer que los participantes del programa tienen la probabilidad del 17.4 \% de mejorar su calidad vida; así como mejorar el estado de salud en un 9.6 \% y en educación aproximadamente en un $15 \%$; Cusacani (2015). En los resultados se puede ver que el mecanismo de focalización es adecuado pues da preferencia a los hogares más pobres, sin embargo, no se encuentra 
evidencia a favor del aumento de matrículas de los niños por sí misma, tampoco se encuentra un impacto significativo en los indicadores de salud infantil, como las puntuaciones z del IMC para la edad y la talla, Gahlaut (2011).

Por otra parte, Díaz y Saldarriaga (2014) dan a conocer que el programa no ha tenido efectos en la salud neo-natal, pero existe cierta evidencia de una mejora en el cuidado pre-natal de las madres gestantes. No obstante, no existe mejora en la salud de los recién nacidos. Además, el programa JUNTOS tuvo un impacto positivo sobre las condicionalidades, el gasto en alimentos, en educación y el estado nutricional; sin embargo, no ha tenido impacto sobre el aprendizaje ni sobre el trabajo infantil. Sánchez \& Rodríguez (2016) Por otra parte, en el estudio realizado por Rodríguez (2017), obtuvo los siguientes resultados, incremento en $26 \%$ de matrículas en niños menores de 17 años afiliados al Programa Juntos y se evidenció aumento de la probabilidad de vacunación de los niños menores de 5 años.

Según Monge, Seinfeld y Campana (2017), el impacto en términos del incremento de los años de educación, asistencia y la reducción de la deserción es positivo y significativo para varones de 15 a 16 años, pero es nulo para las mujeres de la misma edad. Es posible por el tipo de incentivo otorgado por Juntos, que no toma en cuenta diferencias por género de los hijos, asume similares condiciones de demanda educativa para niños y niñas, cuando en realidad la evidencia generada va en contra de este argumento. En cuanto a los resultados en salud, no muestran impactos en términos del parto, incremento de la talla y el peso al nacer. La implementación del programa Juntos se puede medir de dos maneras, en el corto plazo aliviar la pobreza monetaria de un modo focalizado e incrementar al escolar los servicios públicos de salud y educación y en el largo plazo está orientado a preservar la acumulación del capital humano, sentando las bases para romper con la trasmisión generacional de la pobreza Fizbein y Schady, (2009), citado por Sánchez y Rodríguez, (2016).

Por otra parte, Arroyo (2010) refiere que algunas familias utilizan parte del dinero recibido para otras necesidades como es para ahorrar e invertir en mejorar sus viviendas y/o como capital para sus negocios y en otros casos adquirir materiales de trabajo y o productos de primera necesidad para toda la familia. Según la percepción de algunas autoridades se tiene porcentajes elevados de los beneficiarios que no hacen uso adecuado del dinero que reciben del programa.

El propósito del presente estudio fue determinar si el programa produjo los efectos deseados en las personas, hogares e instituciones a los cuales éste se aplica; obtener una estimación cuantitativa de estos beneficios y evaluar si ellos son o no atribuibles a la intervención del programa (Aedo, 2005, citado en Rodríguez, (2017).

Los programas de transferencia monetaria condicionada tienen como propósito aliviar los efectos de la pobreza a través de un subsidio directo al hogar beneficiario con el cumplimiento de corresponsabilidades 
destinadas a mejorar el capital humano, si bien el ingreso adicional debe aumentar el ingreso que dispone el hogar beneficiario, este puede ser retraído por diversos factores no esperados, Velásquez (2017).

En el estudio Impacto del programa social "Juntos" sobre la brecha de la pobreza en el Perú, para el periodo 2013 - 2014. Los resultados sugieren que el programa no ha reducido la brecha de la pobreza en el periodo de análisis, debido a que se encontró serios problemas de focalización en dicho programa. Almeida et al. (2017). El propósito del programa Juntos está orientado a contribuir al alivio de la pobreza y romper con el círculo vicioso de la transmisión intergeneracional de la misma a través de la generación de capital humano en la población más pobre y vulnerable, para ello selecciona a los hogares en situación de pobreza y pobreza extrema de acuerdo a la clasificación socioeconómica del Sistema SISFHO.

En la lucha contra la pobreza, los resultados e impacto logrados fueron: En educación, los estudios cuantitativos y cualitativos revelan incremento de la matrícula del primero al segundo grado de primaria y de la primaria a la secundaria. No se aprecian cambios en la asistencia, ni en la deserción escolar entre los menores de los hogares participantes. Estos resultados son diferenciados según el contexto cultural, en las zonas rurales el impacto es menor que en zonas de menor valoración o con dificultades de acceso como es en las zonas amazónicas. SITEAL (2018).

De acuerdo a Monge et al. (2017) el impacto del Programa Juntos fue: La asignación del subsidio que generó un aumento en el consumo per cápita de los hogares y el incremento de la asistencia a la escuela. Por otro lado, el proceso de matrícula halló un incremento del 96 \% en los niveles de educación inicial, primaria y secundaria. En salud se analizó los registros de asistencia a los establecimientos en las áreas de crecimiento y desarrollo- CRED, cumplieron con la asistencia a las cuatro fechas programadas para su evaluación; pero no se ha alcanzado al $100 \%$, sino al $94.36 \%$ debido al incumplimiento de las metas de crecimiento y desarrollo (vacunación completa, desparasitación, consumo de suplementos vitamínicos otorgados para niños de 5 años) y los controles pre natales de las gestantes, Cerna (2019).

El presente estudio se basó en la teoría del cambio, esta teoría ayudo a comprender cuales son los mecanismos subyacentes entre los procesos y los efectos esporádicos de un determinado programa de intervención que permite conocer cuándo y cómo funciona dicho programa. Algunos investigadores argumentan que la teoría del programa es útil si se le emplea como un mapa para formular las preguntas correctas sobre el programa o intervención que se evalúa Weiss (1997), Chen (1990-2005), citados en Álvarez y Preinfalk (2018).

Por otra parte, la teoría del cambio es la descripción conceptual de los mecanismos de transmisión de efectos que se generan a partir de una intervención en un grupo de la población de manera que se hace evidente los supuestos de funcionamiento y los efectos esperados en cada punto del proceso de ejecución Gertler (2011), citado por Monge et al. (2017). Es decir, los cambios se inician cuando el programa entrega 
los bienes o servicios a su población objetivo y este lo consume o lo usa. El consumo de dichos bienes genera efectos en la población (resultados) a corto y mediano plazo.

\section{Método}

Tipo de estudio. El presente estudio es de tipo aplicado; porque ha sido dirigido a aprovechar los conocimientos existentes obtenidos de la experiencia práctica del servicio del programa social Juntos para la reducción de la pobreza. El enfoque de la investigación fue cuantitativo, porque el procesamiento y estimación de los parámetros del modelo se ha realizado con los programas de Excel y con el software Stata 15. Para detectar los efectos atribuibles al programa Juntos, así mismo se utilizó Diferencias en Diferencias (DIF and DIF) con efectos fijos a nivel del hogar y el individuo.

El nivel del estudio fue explicativo, porque estuvo orientado a determinar el impacto del programa Juntos en la situación de los beneficiarios en lo que respecta a las necesidades básicas insatisfechas, pobreza, salud y educación. El diseño de investigación es cuasi experimental longitudinal, porque se comparó el cambio entre el grupo control y el grupo de tratamiento antes y después de la intervención del programa Juntos, para evaluar los resultados; y es longitudinal porque se recolectó información de aquellos hogares que fueron afiliados desde el año 2015 hasta el 2019. Se midió los efectos físicos a nivel del hogar, individuo tomando en cuenta de la disponibilidad de información de la línea de base y de seguimiento.

Población y muestra. Referente a población, en el presente estudio se tuvo una población muestral de 3990 hogares que tienen características comunes específicas, la muestra para el tratamiento fue 1459 y para el grupo control 1399 hogares de la región Junín, los criterios de inclusión fueron los niños y mujeres pertenecientes al programa 2015-2019 y en el grupo de tratamiento fueron hogares que cumplen con los requisitos de admisión al programa y para el grupo control fueron hogares con características similares al programa y a la población objetiva 2015-2019 y los de exclusión fueron hogares con condiciones económicas óptimas, el tipo de muestreo fue probabilístico aleatorio.

Instrumentos de recolección de datos. La técnica que se aplicó fue la encuesta y el instrumento de recolección de datos fue el cuestionario de vida y pobreza ENAHO-PANEL (2019), que se utilizó en poblaciones grandes. Las variables del estudio fueron: impacto del programa Juntos, necesidades básicas insatisfechas, pobreza, salud y educación. Las dimensiones fueron: vivienda, consumo, horas de trabajo, ocupación, monto de compra, gasto de pobreza, salud y educación, la cantidad de ítems consideradas fueron para las necesidades básicas insatisfechas de los beneficiarios del programa Juntos fue 25 preguntas, para la dimensión de la pobreza se tuvo 9 preguntas, en cuanto a salud de los beneficiarios se 
tuvo 12 preguntas, así mismo para la dimensión de educación se tuvo 7 preguntas, sumando un total de 59 ítems.

Procedimientos de la recopilación de datos. Cabe resaltar, que por las constantes cuarentenas que se tuvo por la Pandemia del COVid-19 durante el año 2020, se tuvo limitaciones para la recolección de datos del trabajo de campo, motivo por lo que el presente estudio fue basado en datos secundarios del Instituto Nacional de Estadística e Informática, de la encuesta nacional de Hogares en condiciones de vida y pobreza ENAHO-PANEL (2015-2019), considerando que los datos fueron validados por el Concejo Consultivo Nacional de Estadística e Informática (CCONEI, 2001). La confiabilidad se calculó de acuerdo al número de casos u observaciones contenidas en la ficha técnica de la Encuesta Nacional de Hogares sobre Condiciones de Vida y Pobreza (2018).

Análisis de datos. Los datos se recolectaron utilizando los microdatos del ENAHO e INEI (2015-2019), se analizó la significancia estadística, según el modelo (Dif and Dif) teniendo en cuenta, ${ }^{* *} p<0.05$, con un poder estadístico significativo, ${ }^{* * *} p<0.01$, alta significancia estadística y bajo poder estadístico el $* p<0.1$ a través de los indicadores de necesidades básicas insatisfechas, pobreza, salud y educación donde se evidenciaron tanto en el grupo control como el de tratamiento los impactos logrados.

\section{Resultado}

En relación a las necesidades básicas insatisfechas, los resultados obtenidos del impacto significativo del Programa Juntos fue vivienda generando un aumento de bajo nivel significativo ( $p$-valor $<0.10$ ) con un aumento de 13.7 \% de desviación estándar con un alumbrado público por generador en su hogar que ocasionó un aumento de bajo nivel significativo ( $p$-valor $<0.10$ ), con un incremento de $0.5 \%$ de desviaciones estándares en el grupo de tratamiento.

La tabla 1, presenta los resultados del análisis de impacto sobre las necesidades básicas insatisfechas observándose, que el programa parece haber afectado al grupo de tratamiento en una magnitud estadísticamente significativa $\mathbf{p}<\mathbf{0 . 0 5}$, no utilizan alumbrado eléctrico en su hogar y en algunos casos solo usan generadores. Además, cabe resaltar, que los beneficiarios del programa juntos quieren trabajar más horas de las que normalmente trabajan por la necesidad apremiante para la subsistencia de su familia. El resultado obtenido por el grupo de tratamiento generó un aumento de alto nivel significativo ( $p$-valor < 0.01 ) con un incremento de 4.3 desviaciones estándares (ver tabla 1 ). 
Tabla 1

Necesidades básicas de los beneficiarios del programa juntos

Dif en Dif- Modelo con efectos aleatorios-

\begin{tabular}{|c|c|c|c|c|}
\hline \multirow[b]{2}{*}{ Variable } & \multirow[b]{2}{*}{ Nombre } & \multirow[b]{2}{*}{$\mathbf{N}$} & \multicolumn{2}{|c|}{ Tratamiento en tratados } \\
\hline & & & Coeficiente & $\begin{array}{l}\text { Error } \\
\text { Estándar }\end{array}$ \\
\hline Tipo de vivienda & p101 & 3990 & $0,137^{*}$ & 0,073 \\
\hline El material predominante en las paredes exteriores es: & p102 & 3990 & $-0,225$ & 0,159 \\
\hline El material predominante en los pisos es: & p103 & 3990 & 0,025 & 0,070 \\
\hline El material predominante en los techos es: & p103a & 3990 & $-0,182 * *$ & 0,091 \\
\hline La vivienda que ocupa su hogar es : & p105a & 4000 & $-0,113$ & 0,113 \\
\hline ¿El agua es potable? & p110a1 & 3327 & $-0,051 *$ & 0,029 \\
\hline Calidad bacteriológica del agua & p110a & 3996 & 0,025 & 0,055 \\
\hline ¿Cuál es el tipo de alumbrado que tiene su hogar? - Electricidad & p1121 & 4000 & $-0,001$ & 0,022 \\
\hline $\begin{array}{l}\text { ¿Cuál es el tipo de alumbrado que tiene su hogar? - } \\
\text { Petróleo/gas (lámpara) }\end{array}$ & p1123 & 4000 & $-0,020 * * *$ & 0,007 \\
\hline ¿Cuál es el tipo de alumbrado que tiene su hogar? - Generador & p1125 & 4000 & $0,005^{*}$ & 0,003 \\
\hline ¿Cuál es el tipo de alumbrado que tiene su hogar? - Otro & p1126 & 4000 & $-0,014$ & 0,012 \\
\hline ¿Cuál es el tipo de alumbrado que tiene su hogar? - No Utiliza & p1127 & 4000 & $0,023 * *$ & 0,009 \\
\hline $\begin{array}{l}\text { ¿Cuál es el combustible que usan en el hogar para cocinar sus } \\
\text { alimentos? - Electricidad }\end{array}$ & p1131 & 4000 & 0,006 & 0,006 \\
\hline Total gasto mensual (s/.) por autoconsumo o autosuministro. & Inp117t4 & 4000 & 0,009 & 0,063 \\
\hline Necesidades Básicas Insatisfechas (1) - Vivienda inadecuada & nbi1 & 4000 & $-0,042 * *$ & 0,020 \\
\hline $\begin{array}{l}\text { Necesidades Básicas Insatisfechas ( } 2 \text { ) - Vivienda con } \\
\text { hacinamiento }\end{array}$ & nbi2 & 4000 & $-0,008$ & 0,017 \\
\hline $\begin{array}{l}\text { Necesidades Básicas Insatisfechas (3) - Hogares con vivienda sin } \\
\text { servicios higiénicos }\end{array}$ & nbi3 & 4000 & $-0,023$ & 0,028 \\
\hline $\begin{array}{l}\text { Necesidades Básicas Insatisfechas (5) - Hogares con alta } \\
\text { dependencia económica }\end{array}$ & nbi5 & 4000 & $-0,002$ & 0,005 \\
\hline $\begin{array}{l}\text { Normalmente, ¿Cuántas horas trabaja a la semana en todas sus } \\
\text { ocupaciones? }\end{array}$ & p520 & 62 & 0,000 & - \\
\hline ¿Quería trabajar más horas de las que normalmente trabaja? & p521 & 1431 & $0,043 * * *$ & 0,015 \\
\hline En su ocupación principal a Ud. le pagan: & p523 & 435 & 0,000 & - \\
\hline ¿Cuánto fue el monto total de la compra? & Inp601c & 4000 & $-0,002$ & 0,037 \\
\hline
\end{tabular}

Nota. ${ }^{* * *} \mathrm{p}<0.01,{ }^{* *} \mathrm{p}<0.05,{ }^{*} \mathrm{p}<0.1$. Los datos son proporcionados por el Instituto Nacional de estadística e informática (INEI, 2019)

\section{Pobreza}

En relación a la situación de pobreza, los resultados obtenidos tanto para el corto como para el mediano plazo, evidenciaron un impacto significativo en vista que el programa generó aumentos pequeños de moderado nivel significativo ( $p$-valor $<0.05$ ), con un incremento de $5.9 \%$ desviaciones estándares en términos de pobreza en el grupo de tratamiento, (ver tabla 2). 
Tabla 2

Pobreza de los beneficiarios del programa juntos

Dif en Dif- Modelo con efectos aleatorios-

\begin{tabular}{|c|c|c|c|c|}
\hline \multirow[b]{2}{*}{ Variable } & \multirow[b]{2}{*}{ Nombre } & \multirow[b]{2}{*}{$\mathbf{N}$} & \multicolumn{2}{|c|}{ Tratamiento en tratados } \\
\hline & & & Coeficiente & $\begin{array}{c}\text { Error } \\
\text { Estándar }\end{array}$ \\
\hline $\begin{array}{l}\text { ¿El gasto realizado fue: Cubierto por el seguro? : Control de salud de } \\
\text { los niños }\end{array}$ & p4157_10 & 448 & 0,000 & - \\
\hline Hogares con niños que no asisten a la escuela & nbi4 & 4000 & 0,002 & 0,006 \\
\hline $\begin{array}{l}\text { ¿Estuvo trabajando o realizando alguna tarea en el hogar o fuera de } \\
\text { él? - } 5 \text { a } 17 \text { años de edad }\end{array}$ & $\mathrm{p} 210$ & 1274 & $-0,026$ & 0,043 \\
\hline $\begin{array}{l}\text { ¿Qué labores o tareas realizó en el hogar o fuera de él? - } 5 \text { a } 17 \text { años } \\
\text { de edad }\end{array}$ & $\mathrm{p} 211 \mathrm{a}$ & 1169 & $0,664^{*}$ & 0,364 \\
\hline ¿Cuántas horas en total realizó estas tareas? & p211d & 252 & 1,959 & 3,683 \\
\hline Pobreza & pobreza & 4000 & $0,059 * *$ & 0,030 \\
\hline Gasto total per capita & gastalpk & 3506 & 0,080 & 0,085 \\
\hline Gasto total por hogar per capita & gasthogpk & 3998 & 0,060 & 0,052 \\
\hline Severidad & severi & 4000 & $-284,816$ & 191,494 \\
\hline Brecha de Pobreza & brecha & 4000 & 2,913 & 11,207 \\
\hline
\end{tabular}

Nota. ${ }^{* * *} \mathrm{p}<0.01,{ }^{*} \mathrm{p}<0.05,{ }^{*} \mathrm{p}<0.1$ Los datos son proporcionados por el Instituto Nacional de estadística e informática (INEI, 2019)

\section{Salud}

En la tabla 3, se evidenció los impactos del programa social Juntos en los indicadores de salud, en cuanto al cumplimiento del esquema de vacunación que exige el Ministerio de Salud (MINSA), pareciera mostrar un incremento estadísticamente significativo negativo $-p<0.1$, esto resulta mejor en el grupo de control que en el grupo de tratamiento; es posible también concluir la ocurrencia de impacto significativo negativo sobre estas variables debido a que el programa solo condiciona al hogar a acudir a su cita para los controles de CRED. Cabe mencionar que el proceso de inmunización en los niños depende en gran medida de la disponibilidad de oferta de los centros de salud, lo cual escapa al cumplimiento de las responsabilidades por el personal de salud con un incremento de $5.2 \%$ de desviación estándar en el grupo control. Se observa también un efecto significativo negativo $-p<0.1$, en algún miembro de la familia del grupo control que se encuentra con alguna enfermedad o malestar crónico.

En general, éstos resultados indican que en el programa Juntos no se encontró un resultado significativo del grupo de tratamiento en comparación con el grupo control en los indicadores de suplemento de hierro, control de crecimiento del niño, seguro de controles por embarazo, seguro de atención de parto y en planificación familiar mujeres de 12 a 49 años de edad, (ver tabla 3). 


\section{Tabla 3}

Salud de los beneficiarios del programa juntos

\begin{tabular}{|c|c|c|c|c|}
\hline \multirow[b]{3}{*}{ Variable } & \multicolumn{4}{|c|}{ Dif en Dif- Modelo con efectos aleatorios- } \\
\hline & \multirow[b]{2}{*}{ Nombre } & \multirow[b]{2}{*}{$\mathbf{N}$} & \multicolumn{2}{|c|}{ Tratamiento en tratados } \\
\hline & & & Coeficiente & $\begin{array}{l}\text { Error } \\
\text { Estándar }\end{array}$ \\
\hline $\begin{array}{l}\text { ¿Ha efectuado consulta? - Programa de vacunas } \\
\text { (inmunizaciones) }\end{array}$ & $\mathrm{p} 413 \mathrm{~b} 2$ & 3734 & $-0,004$ & 0,024 \\
\hline ¿Cómo lo obtuvo el Programa de vacunas? & $\mathrm{p} 413 \mathrm{~b} 2 \mathrm{a}$ & 734 & $-0,103 *$ & 0,054 \\
\hline $\begin{array}{l}\text { ¿Ha efectuado consulta? - Suplemento de hierro (gestantes y } \\
\text { niños menores de } 3 \text { años de edad) }\end{array}$ & p413d2 & 1051 & 0,017 & 0,068 \\
\hline ¿Cómo lo obtuvo el Control de Crecimiento del niño? & p413b1a & 444 & 0,000 & - \\
\hline $\begin{array}{l}\text { Logaritmo de ¿El gasto realizado fue: Cubierto por el seguro? : } \\
\text { Controles por embarazo }\end{array}$ & Inp4157_15 & 4000 & 0,000 & - \\
\hline $\begin{array}{l}\text { Logaritmo de ¿El gasto realizado fue: Cubierto por el seguro? : } \\
\text { Atenciones de parto }\end{array}$ & Inp4157_16 & 4000 & 0,000 & - \\
\hline Controles por embarazo & p414_15 & 3734 & 0,002 & 0,003 \\
\hline Atenciones de parto & p414_16 & 3734 & 0,001 & 0,002 \\
\hline ¿Padece de alguna enfermedad o malestar crónico? & p401 & 3734 & $-0,052^{*}$ & 0,029 \\
\hline $\begin{array}{l}\text { ¿Ha efectuado consulta? - Planificación Familiar (mujeres de } \\
12 \text { a } 49 \text { años de edad) }\end{array}$ & p413d1 & 1051 & 0,020 & 0,038 \\
\hline
\end{tabular}

Nota. ${ }^{* * *} \mathrm{p}<0.01, * * \mathrm{p}<0.05, * \mathrm{p}<0.1$. Los datos son proporcionados por el Instituto Nacional de estadística e informática (INEI, 2019).

\section{Educación}

En relación a educación, los resultados obtenidos tanto para el corto como para el mediano plazo, evidenciaron que el programa social Juntos, generó aumentos pequeños de moderado nivel significativo $(p<0.05)$, con un incremento de $7.0 \%$ desviaciones estándares en estudiantes que están matriculados en algún centro o programa de educación básica o superior. En cuanto a la matrícula de los niños, niñas y adolescentes en algún centro o programa de educación básica o superior, cumplen su compromiso de matricularse. Por otro lado, se evidenciaron aumentos pequeños con bajo nivel de significancia, en el idioma o lengua materna que aprendió en su niñez $(p<0.10)$. Referente al idioma o lengua materna, tiene un impacto positivo $0.106^{*}$ significativo al $10 \%$, el grupo de tratamiento, está mejor que el grupo control en cuanto al aprendizaje del idioma. Así mismo la pensión mensual de enseñanza, fue significativamente positivo en el grupo control $-0.044^{* *}$ y es significativo al $5 \%$, porque tienen mejores posibilidades económicas para hacer efectivo sus pensiones que el grupo de tratamiento. Es decir, los resultados obtenidos en educación tanto para el corto como para el mediano plazo, evidenciaron que el programa generó aumentos pequeños de moderado nivel significativo ( $p$-valor $<0.05$ ), con un incremento de7.0 \% desviaciones estándares en la matrícula de algún centro o programa de educación básica o superior, (ver tabla 4). 


\section{Tabla 4}

Educación de los beneficiarios del programa juntos

Dif en Dif- Modelo con efectos aleatorios-

\begin{tabular}{|c|c|c|c|c|}
\hline \multirow[b]{2}{*}{ Variable } & \multirow[b]{2}{*}{ Nombre } & \multirow[b]{2}{*}{$\mathbf{N}$} & \multicolumn{2}{|c|}{ Tratamiento en tratados } \\
\hline & & & Coeficiente & $\begin{array}{c}\text { Error } \\
\text { Estándar }\end{array}$ \\
\hline ¿Cuál es el último año o grado de estudios y nivel que aprobó? - Año & p301b & 2622 & $-0,174$ & 0,126 \\
\hline ¿Cuál es el idioma o lengua materna que aprendió en su niñez? & p300a & 3210 & $0,106 *$ & 0,064 \\
\hline ¿Sabe leer y escribir? & p302 & 1717 & $-0,016$ & 0,043 \\
\hline ¿El resultado que obtuvo el año pasado (...) fue? & p305 & 1437 & 0,059 & 0,143 \\
\hline $\begin{array}{l}\text { ¿Está matriculado en algún centro o programa de educación básica o } \\
\text { superior? }\end{array}$ & p306 & 3210 & $0,070 * *$ & 0,031 \\
\hline $\begin{array}{l}\text { ¿Recibió enseñanza en algún centro o programa de estudios cuya } \\
\text { duración sea menos a } 3 \text { años? }\end{array}$ & p310 & 3210 & $0,036^{*}$ & 0,020 \\
\hline $\begin{array}{l}\text { ¿Cómo obtuvieron el (la)? Pensión Mensual de Enseñanza - } \\
\text { Programa Social }\end{array}$ & p3121a5 & 400 & $-0,044 * *$ & 0,019 \\
\hline
\end{tabular}

\section{Discusión}

\section{Necesidades básicas insatisfechas}

En relación a las necesidades básicas insatisfechas, los resultados obtenidos de impacto significativo del Programa Juntos, únicamente fue vivienda generando un aumento de bajo nivel significativo ( $p$-valor < 0.10), con un aumento de $13.7 \%$ de desviación estándar con un alumbrado público por generador en su hogar que ocasionó un aumento de bajo nivel significativo ( $p$-valor $<0.10$ ), con un incremento de $0.5 \%$ de desviaciones estándares en el grupo de tratamiento. Además, los beneficiarios del programa juntos quieren trabajar más horas de las que normalmente trabajan por la necesidad apremiante para la subsistencia de su familia, los resultados obtenidos por el grupo de tratamiento generaron un aumento de alto nivel significativo ( $p$-valor $<0.01$ ) con un incremento de $4.3 \%$ de desviaciones estándares.

Estos resultados se sustentan en Arroyo (2010) cuando refiere que algunas familias utilizan parte del dinero recibido para otras necesidades como es para ahorrar e invertir en mejorar sus viviendas y/o como capital para sus negocios y en otros casos adquirir materiales de trabajo y o productos de primera necesidad para toda la familia. 


\section{Pobreza}

Un hallazgo interesante en cuanto al indicador de pobreza es, los resultados obtenidos tanto para el corto como para el mediano plazo, evidenciaron un impacto significativo en vista que el programa generó aumentos pequeños de moderado nivel significativo ( $p$-valor $<0.05$ ), con un incremento de $5.9 \%$ desviaciones estándares en términos de pobreza en el grupo de tratamiento. Este hallazgo fue respaldado por Perova y Vakis (2010) cuando mencionan que los resultados cuantitativos encuentran impactos positivos en varias dimensiones de bienestar, tales como: la reducción de pobreza, mejora de ingreso, salud y educación. Sin embargo, la evaluación no encuentra impactos en indicadores finales como lo es la nutrición infantil, anemia, desarrollo cognitivo. No obstante, de acuerdo a la información de la ENAHO (2019) para los años 2013 y 2014 no se observa mejoras en la brecha de pobreza a través del tiempo.

Por otra parte, según los resultados hallados de Fiszbein y Schady (2009) citado por Monge et al. (2017) manifiestan que los programas sociales de transferencias condicionados para varios países de Latinoamérica, encuentran reducciones en los niveles de pobreza. También concuerda con el Banco Mundial BM (s/f) citado por Aramburu (2010) encuentra que la transferencia monetaria del programa equivale al $13 \%$ del gasto en consumo mensual (que incluye gasto monetario más la valorización del autoconsumo) este incremento permite reducir en $5 \%$ la brecha de la pobreza (distancia monetaria entre el valor del consumo y la línea de pobreza). Del mismo modo Juntos tiene un impacto positivo sobre el consumo de hogares impulsado por el incremento en el rubro de alimentos (34 \%). En síntesis, Juntos reduce la brecha de la severidad de la pobreza en $5 \%$ y $6 \%$ respectivamente. Así mismo, la magnitud del impacto sobre las brechas de pobreza es consistente con experiencias de otros países. Sin embargo, cabe resaltar que la suma transferida por el programa juntos, es insuficiente para lograr que los hogares participantes en el programa superen la línea de pobreza teniendo una diversidad de necesidades. Además, se sostiene en Almeida et al. (2017) cuando establecen que el Programa de Transferencias condicionados Juntos no ha reducido la brecha de pobreza a través del tiempo. Es decir, no se ha logrado el impacto debido a que el monto bimestral de $S / 200.00$ que se entrega es insuficiente. Se coincide con los resultados obtenidos por Jaramillo y Sánchez (2012) cuando concluyen que "posiblemente los más pobres entre los pobres se estarían beneficiando menos", esto implica que las familias beneficiarias que se encuentran en situación de pobreza extrema no estarían logrando mejoras.

Por otra parte, el resultado se respalda en Monge et al. (2017), cuando manifiestan que el Programa Juntos no muestra impactos en términos de pobreza y pobreza extrema; pero si sobre la severidad de la pobreza, la brecha y severidad de la pobreza extrema en $2.7 p p, 3.4 p p$ y $2.7 p p$ respectivamente. Los resultados parecen indicar que el programa habría logrado incrementar levemente el gasto y la calidad de gasto de los hogares, pero sin que ello implique reducir la tasa de la pobreza o de pobreza extrema. Así mismo, se sostiene en Velásquez (2017) cuando expresa que el Programa Juntos tuvo impacto positivo 
sobre el ingreso total del hogar beneficiado, es decir los hogares beneficiados incrementaron sus ingresos alrededor del 20 \% más en relación a los hogares que no recibieron la transferencia económica; que puede ser por la adecuada administración de los recursos económicos para el hogar y la utilización en actividades complementarias como fuente de ingresos alternativas. Esto refleja que la transferencia representa una fuente de ingresos muy importante para la economía familiar de los hogares con ingresos económicos precarios.

Además, estos resultados se respaldan en Melchor y Rodríguez (2019) cuando manifiestan que el Programa Juntos a nivel de la región Junín contribuyó de manera significativa en la disminución de la pobreza en Junín en un 20 \% en el 2016 y en los últimos años no se percibe impacto significativo en la población beneficiaria.

\section{Salud}

Los impactos del programa en los indicadores de salud, en cuanto al cumplimiento del esquema de vacunación que exige el MINSA , pareciera mostrar un incremento estadísticamente significativo negativo $-p<0.1$, esto resulta mejor en el grupo de control que en el grupo de tratamiento; es posible también concluir la ocurrencia de impacto significativo negativo sobre estas variables debido a que el programa solo condiciona al hogar a acudir a su cita para los controles de CRED y cabe mencionar que el proceso de inmunización en los niños depende en gran medida de la disponibilidad de oferta de los centros de salud, lo cual escapa al cumplimiento de las responsabilidades por el personal de salud con un incremento de $5.2 \%$ de desviación estándar en el grupo control. Se observa un efecto estadísticamente significativo negativo $-p<0.1$, es decir que algún miembro de la familia del grupo control se encuentra con alguna enfermedad o malestar crónico. Al parecer para la muestra considerada el Programa Juntos no habría tenido ningún efecto esperado en los indicadores de suplemento de hierro, control de crecimiento del niño, controles de embarazo y atenciones de parto.

El estudio actual encontró el impacto en los indicadores de salud que se sostienen en Monge et al. (2017) cuando concluyen que la ocurrencia de impactos significativos sobre estas variables es debido a que el programa sólo condiciona al hogar a acudir a su cita para los controles CRED, pero la vacunación de los niños depende en gran medida de la disponibilidad de oferta de vacunas en los establecimientos de salud, lo cual escapa al cumplimiento de las corresponsabilidades. Además, se sustenta en el Sistema de Información de Tendencias en América Latina (SITEAL,2010) cuando expresa que la asistencia a controles de crecimiento y desarrollo (CRED) tienen una probabilidad de 37 puntos porcentuales mayor que los del grupo de control y de 2.2 puntos porcentuales para recibir atención médica cuando lo necesitan, pero tiene una probabilidad de solo 7 puntos porcentuales mayor; sin embargo, la misma fuente aclara que las metas de acceso universal a los servicios básicos de salud están aún muy lejanas. También en el estudio 
actual de Cavero et al. (2017) no se encontraron resultados significativos en la mayoría de indicadores de salud prenatal ni el estado nutricional infantil, el programa evaluó 22 indicadores, sin embargo, no se hallaron efectos en la mayoría de ellos. En la cadena causal relacionada con la salud prenatal, no hubo efectos en el número de controles prenatales, edad del primer control ni si realizaron su primer control en el primer trimestre. Por tanto, tampoco en parto institucional, incremento de la talla al nacer, o peso al nacer. En el primer caso la ausencia de efectos podría deberse a los mayores costos de desplazamiento de las gestantes o a que las coberturas podrían ser altas. En la cadena causal vinculada con la salud infantil, a diferencia de otros autores se hallaron efectos significativos en el cumplimiento de las pautas de los controles más no en el número de controles por grupo etario y efectos no robustos en el cumplimiento del esquema de vacunación.

Este hallazgo es apoyado ampliamente por Rodríguez (2017) cuando evidencia un incremento en la probabilidad de vacunación de los niños menores de 5 años; además existe un aumento de la probabilidad de mejora en el índice de masa corporal, de igual manera se muestra la probabilidad de aumento del valor de hemoglobina para niños menores de 5 años. En salud analizó los registros de asistencia a los establecimientos en las áreas de crecimiento y desarrollo (CRED), donde expone que cumplieron con la asistencia a las cuatro fechas programadas para su evaluación; pero no se ha alcanzado al 100 \%, sino al 94.36 \% debido al incumplimiento de las metas de crecimiento y desarrollo (vacunación completa, desparasitación, consumo de suplementos vitamínicos otorgados para niños de 5 años) y los controles pre natales de las gestantes. Cerna (2019)

Por otra parte, se respalda en Benel et al. (2018) cuando hallaron en el distrito de Acolla que el Programa JUNTOS cumplen con las corresponsabilidades que exige el Programa referidos a que los niños y niñas no dejen de asistir a la escuela, las madres embarazadas asistan a sus controles prenatales, y los niños recién nacidos reciban sus vacunas y asistan a sus controles de crecimiento y desarrollo.

\section{Educación}

En relación a educación los resultados obtenidos tanto para el corto como para el mediano plazo, evidenciaron que el programa generó aumentos pequeños de moderado nivel significativo ( $p$-valor < 0.05), con un incremento de $7.0 \%$ de desviaciones estándares que está matriculado en algún centro o programa de educación básica o superior. Así mismo se evidenciaron aumentos pequeños con bajo nivel de significancia en el idioma o lengua materna que aprendió en su niñez ( $p$-valor $<0.10)$.

Los resultados se respaldan en Vakis y Perova (2009) citado por Aramburu (2010) cuando evidencia que el Programa social Juntos ha logrado un impacto en el incremento de la matrícula en 4 puntos porcentuales entre los menores de 6 a 14 años en los distritos de intervención frente a los de control, pero no se registran incrementos en la asistencia escolar al comparar estos dos grupos. Cabe resaltar que 
la matrícula se da en los primeros grados alcanzando en el grupo de tratamiento una matrícula del $93 \%$ frente al 83 \% del grupo control; es decir se evidencia una mejora en la matrícula, pero no tienen pruebas en cuanto a la asistencia y deserción y tampoco se demuestra que es un logro del Programa Juntos. Así mismo se sostiene en Galasso (2007) citado por Monge et al. (2017) cuando señalan que la probabilidad de la matrícula para niños entre 6 y 15 años se incrementa en 7 pp. Además, la tasa de matrícula se incrementó en 1.3pp en zonas urbanas y 2.95 en zonas rurales para los niños entre 8 y 13 años, mientras que para jóvenes entre 14 y 17 años, ésta tasa se incrementó en 5.3pp en zonas urbanas y $5 p p$ en zonas rurales, Atanasio y otros (2005) citado por Monge et al. (2017). Así mismo el Programa Juntos incrementó la tasa de matrícula en general, teniendo mayor efecto para 5to y 6 grado de primaria, así como 1ro y 2do de secundaria. Mesinas (2010) citado por Monge et al. (2017). También, se respalda en Rodríguez (2017) cuando halló un incremento en $26 \%$ de matrículas en niños menores de 17 años afiliados al Programa Juntos.

Por otro lado, se sostiene en IPE-UNESCO (2018) cuando revelan un incremento de la matrícula del primero al segundo grado de primaria y de la primaria a la secundaria. No se aprecian cambios en la asistencia, ni en la deserción escolar entre los menores de los hogares participantes. Estos resultados son diferenciados según el contexto cultural en las zonas rurales el impacto es menor que en zonas de menor valoración o con dificultades de acceso como en las zonas amazónicas. Finalmente, el proceso de matrícula halló un incremento del 96 \% en los niveles de educación inicial, primaria y secundaria (Cerna, 2019). Por otra parte, las usuarias que participaron en el análisis cualitativo piloto de la provincia de Huamanga; y Luricocha en la provincia de Huanta, la mayoría tienen como lengua materna el quechua; solo dos mujeres tenían el castellano como idioma nativo. La mayoría de las mujeres comprenden el castellano, pero se les hace difícil comunicarse en esta lengua. (Alcázar y Espinoza, 2014).

\section{Conclusiones}

En el estudio de cuatro años del programa Juntos del 2015 al 2019, basados en datos secundarios, las conclusiones fueron los siguientes:

El impacto del Programa social Juntos en las necesidades básicas insatisfechas fue de bajo nivel significativo, los hallazgos pueden ayudarnos a comprender que el indicador más impactante fue que no cuentan con alumbrado eléctrico en sus hogares y solo algunos utilizan generadores a motor. Así mismo se evidenció que los padres de familia del grupo de tratamiento trabajan más de 8 horas para cubrir sus necesidades básicas.

El resultado de este estudio en pobreza evidenció un impacto de bajo nivel significativo, es decir hay una disminución muy leve de la pobreza en el grupo de tratamiento comparado al grupo control. 
El impacto del Programa social Juntos en salud fue negativo, porque los hallazgos no fueron muy alentadores, éstos resultados indican que no se encontró un resultado significativo del grupo de tratamiento en comparación con el grupo control en los indicadores de suplemento de hierro, control de crecimiento del niño, seguro de controles por embarazo, seguro de atención de parto y en planificación familiar mujeres de 12 a 49 años de edad, pero hay algunas conclusiones confiables de impacto significativo negativo en dos aspectos principales en la estrategia de vacuna y en si padece de alguna enfermedad o malestar crónico . El programa solo condiciona al hogar a acudir a su cita para los controles de CRED y el proceso de inmunización en los niños que depende en gran medida de la disponibilidad de oferta de los centros de salud, lo cual escapa al cumplimiento de las responsabilidades por el personal de salud y el otro aspecto destaca las enfermedades o malestar crónico de algún miembro de la familia del grupo control.

Los resultados obtenidos en el aspecto de educación, son de bajo nivel significativo porque los datos indican que se incrementó solo la matrícula en la educación inicial, primaria y secundaria; no siendo alentadores en el saber leer, escribir y en el idioma o lengua nativa. Por lo tanto, se puede suponer que el programa social Juntos no logró el impacto deseado en el grupo de tratamiento.

Estos hallazgos, sin duda demuestran que el programa social Juntos en la región Junín no ha tenido un impacto significativo en los beneficiarios, siendo de bajo nivel significativo en las necesidades básicas insatisfechas, en pobreza fue de moderado nivel significativo al igual que en educación y en salud el impacto fue significativo negativo. Sin embargo, el impacto del programa social Juntos debería ser analizado a mayor profundidad, debido a que hay algunas conclusiones donde el programa social Juntos evidenció un impacto moderadamente significativo, es decir hay una disminución leve de la severidad de pobreza en el grupo de tratamiento en relación al grupo control, pero no explica la ocurrencia del bajo nivel de impacto en salud, educación y necesidades básicas insatisfechas. Por lo tanto, se recomienda realizar estudios cualitativos de las variables mencionadas.

\section{Referencias}

Alcázar, L., y Espinoza K. (2014). Metodologías de investigación y evaluación de políticas y programas sociales. Grupo de análisis para el desarrollo, (19), 1-104. https://www.grade.org.pe/wpcontent/uploads/Al19.pdf

Almeida, E., Espinoza, S., Márquez, M., y Panda, M. (2017). Impacto del programa Social "Juntos" sobre la brecha de la pobreza en el Perú, 2013-2014. Horizonte económico, (6), 1- 12. https://fce.unac.edu.pe/images/investigacion/unidad-investigacion/revista/he-n6/a64.pdf 
Álvarez, J., y Preinfalk, M., (2018). Teoría del programa y Teoría del Cambio en la Evaluación para el Desarrollo: Una revisión teórica, práctica. Revista ABRA, 38(56), 1-16. https://doi.org/10.15359/abra.38-56.2

Arroyo, J. (2010) Estudio cualitativo de los efectos del programa Juntos en los cambios de comportamiento de los hogares beneficiarios en el distrito de Chuschi. Ministerio de economía y finanzas. https://www.mef.gob.pe/contenidos/pol_econ/documentos/Estudio_Cualitativo_de_CHUSCHI. pdf

Benel, R., Espinoza, L., y Misari, J., (2018). Prácticas que inciden en la calidad de vida de las familias usuarias del programa juntos en el distrito de Acolla - Jauja. [Tesis de posgrado, Pontificia Universidad Católica del Perú]. Repositorio institucional de la PUCP. http://tesis.pucp.edu.pe/repositorio/handle/20.500.12404/12994

Cavero, D., Cruzado, V., y Cuadra, G. (2017). Los efectos de los programas sociales en la salud de la población en condición de pobreza: evidencias a partir de las evaluaciones de impacto del presupuesto por resultados a programas sociales en Perú. Revista Peruana de Medicina Experimental y Salud Publica, 34(3), 528-37. https://dx.doi.org/10.17843/rpmesp.2017.343.3063

Cardozo, M. (2006) Políticas de lucha contra la pobreza en México. Principales resultados y limitaciones. Revista Venezolana de Sociología y Antropología, 16(45), 15-56. https://www.redalyc.org/pdf/705/70504503.pdf

Cerna, R. (2019). Incidencia del programa nacional del apoyo directo a los más pobres juntos, sobre la deserción escolar y acceso a los servicios de salud de las familias usuarias del programa juntos del distrito de Recuay, años 2011- 2013. [Tesis de posgrado, Universidad Nacional Santiago Antúnez de Mayolo]. Repositorio institucional de la UNASAM. http://repositorio.unasam.edu.pe/handle/UNASAM/3589

Cusacani, R. (2017). Evaluación de impacto del programa nacional de apoyo directo a los más pobres (JUNTOS) en la provincia El Collao-Puno, 2015[Tesis de pregrado, Universidad del Altiplano]. Repositorio institucional de la UNAP. http://repositorio.unap.edu.pe/handle/UNAP/3992

Díaz, J. y Saldarriaga, V. (2014). Efectos del Programa de Transferencias Condicionadas Juntos en el peso al nacer de los niños. Consorcio de investigación económica y social. Informe final. http://cies.org.pe/sites/default/files/investigaciones/informe-final-efecto.pdf

Instituto Nacional de Estadística e Informática, INEI-ENAHO (2015- 2019) Microdatos, base de datos, consulta por encuestas, metodología actualizada, condiciones de vida y pobreza. ENAHO PANEL. 
http://iinei.inei.gob.pe/microdatos/

Decreto Supremo N 106- 2001-PCM. Reglamento del Consejo Consultivo Nacional de Estadística e Informática. (14 de setiembre de 2001).

https://www.inei.gob.pe/media/MenuRecursivo/plan_estadistico_nacional/cconeireglamento.p df

Instituto Nacional de Estadística e Informática, INEI (2019) Encuesta Nacional de Hogares sobre condiciones de Vida y pobreza. Recuperado de la base de datos del Sistema de digitación virtual de investigaciones estadísticas.

https://webinei.inei.gob.pe/anda_inei/index.php/catalog\#_r=1625848416961\&collection=\&cou ntry=\&dtype=\&from=2015\&page=1\&ps=\&sk=\&sort_by=proddate\&sort_order=desc\&to=2019\&t opic $=\&$ view $=s \& v k=j u n t o s$

Gahlaut, A. (2011). An Analysis of the Juntos Cash-Transfer Programme in Peru, with special emphasis on child outcomes. Young Lives student paper,1-32. https://www.younglives.org.uk/sites/www.younglives.org.uk/files/YLSP_Gahlaut_MPhilDissertation_Augl2011.pdf

Sistema de Información de Tendencias Educativas en América Latina SITEAL. (2018). Informe Compilatorio: El PROGRAMA Juntos, Resultados y Retos". En la lucha contra la pobreza. El Perú avanza. https://siteal.iiep.unesco.org/sites/default/files/sit_accion_files/pe_8063.pdf

Jaramillo, M., \& Sánchez, A. (2011). Impacto del programa Juntos sobre nutrición temprana. (Documento de investigación N61). Grupo de Análisis para el desarrollo. http://www.grade.org.pe/upload/publicaciones/archivo/download/pubs/ddt61.pdf

Matías, A. (27 abril de 2019). En Junín hay más de 4 mil nuevos pobres desde 2015. Diario correo.pe https://www.ipe.org.pe/portal/wp-content/uploads/2019/05/2019-04-27-En-Jun\%C3\%ADn-haym\%C3\%A1s-de-4-mil-nuevos-pobres-desde-2015-Informe-IPE-Correo-Huancayo.pdf

Melchor, K. y Rodríguez, Y. (2019) Efectos del Programa Nacional Juntos en la disminución de la pobreza en la región Junín. [Tesis de pregrado, Universidad Nacional del Centro del Perú]. Repositorio institucional de la UNCP. http://repositorio.uncp.edu.pe/bitstream/handle/UNCP/5797/T010_70134772_T.pdf?sequence $=1 \&$ isAllowed $=\mathrm{y}$

Monge, A., Seinfeld, J. y Campana (2017). Informe de Evaluación de Impacto del programa JUNTOS (Resultados finales). Ministerio de Economía y Finanzas. http://evidencia.midis.gob.pe/wp-content/uploads/2018/05/Informe_Final_13.pdf 
Perova, E.; Vakis, R. (2010). El impacto y potencial del Programa JUNTOS en PERÚ: Evidencia de una evaluación no-experimental. (Informes técnicos). Ministerio de Educación. https://repositorio.minedu.gob.pe/handle/20.500.12799/3974

Rodríguez, W., (2017). El Impacto del Programa Juntos en Cajamarca: Una evaluación cuasi experimental para el caso de las poblaciones pobres del distrito de Chetilla [Tesis de pregrado, Universidad Nacional de Cajamarca]. Repositorio institucional de la Universidad Nacional de Cajamarca https://repositorio.unc.edu.pe/bitstream/handle/UNC/1886/Tesis\%20WRZ.pdf?sequence=1\&is Allowed=y

Sánchez, A. y Rodríguez, M. (2016). Atención y educación de la primera infancia en el Perú: GRADE. Investigación para el desarrollo en el Perú: once balances (pp.207-248) GRADE. https://www.grade.org.pe/wp-content/uploads/balanceJUNTOS_AS_35.pdf

Sánchez, A. y Jaramillo, M. (2012). Impacto del Programa Juntos sobre la nutrición temprana. Revista Estudios Económicos, (23) ,53-66.

https://www.bcrp.gob.pe/docs/Publicaciones/Revista-Estudios-Economicos/23/ree-23-sanchezjaramillo.pdf

Sistema de información de Tendencias Educativas en América Latina. (2018). Informe compilatorio: “EI Programa JUNTOS, Resultados y Retos". En la lucha contra la pobreza. Recuperado de SITEAL. https://siteal.iiep.unesco.org/sites/default/files/sit_accion_files/pe_8063.pdf

Velásquez, Y. (2017). Impacto de la transferencia monetaria condicionada del PROGRAMA JUNTOS sobre el ingreso del hogar beneficiario en el Perú, 2013 - 2015 [Tesis pregrado, Universidad Nacional del Altiplano]. Repositorio institucional de la UNA. http://repositorio.unap.edu.pe/bitstream/handle/UNAP/4902/Velasquez_Alanoca_Yosi_Miguel. pdf?sequence=1\&isAllowed $=y$

\section{Contribución de los autores}

EFA, JCPT, ERHG, LMVB, CYBM: El impacto del Programa Juntos en necesidades básicas insatisfechas, pobreza, salud y educación en la región Junín-Perú.

Fuentes de financiamiento

El estudio fue autofinanciado.

Conflictos de interés

No presenta conflicto de intereses.

\section{Correspondencia}

Ivilcas@uncp.edu.pe 